\title{
Respiratory microbiome profiles differ by recent hospitalization and nursing home residence in patients on mechanical ventilation
}

\section{Min-gyung Baek}

Department of Public Health, Korea University

\section{Seongji Woo}

Institute of New frontier Research,

Nam-Eun Kim

Department of Public Health Science, Graduate School of Public Health

\section{Chaeyun Baek}

Department of Public Health

\section{Sungho Won}

Interdisciplinary Program of Bioinformatics

\section{Youngmi Kim}

Institute of New frontier Research

Jae Jun Lee

Institute of New frontier Research

Hana Yi

School of Biosystems and Biomedical Sciences

Ji Young Hong ( $\nabla$ mdhong@hallym.or.kr)

Chuncheon Sacred Heart Hospital https://orcid.org/0000-0002-3132-7706

\section{Research}

Keywords: HCAP, microbiome, pneumonia, mechanical ventilation

Posted Date: September 1st, 2020

DOI: https://doi.org/10.21203/rs.3.rs-63988/v1

License: (9) (i) This work is licensed under a Creative Commons Attribution 4.0 International License. Read Full License

Version of Record: A version of this preprint was published on December 7th, 2020. See the published version at https://doi.org/10.1186/s12967-020-02642-z. 


\section{Abstract}

Background: Healthcare-associated pneumonia (HCAP) is a heterogeneous disease. We redefined nursinghome- and hospital-associated infections (NHAl) group by revising existing HCAP risk factors. The NHAI group comprised nursing home residents with a poor functional status, or recent (past 90 days) hospitalization or recent (past 180 days) antibiotic therapy. Our aim was to determine whether respiratory microbiota profiles are related to newly defined NHAI group in critically ill patients on mechanical ventilation.

Methods: The 180 endotracheal aspirates (ETAs) from 60 mechanically ventilated ICU patients (NHAI group, $\mathrm{n}=24$; non-NHAI group, $\mathrm{n}=36$ ) were prospectively collected on days 1, 3 and 7 in a university hospital. The bacterial community profiles of the ETAs were explored by $16 \mathrm{~S}$ rRNA gene sequencing. A phylogenetic-treebased microbiome association test (TMAT), generalized linear mixed models (GLMMs), the Wilcoxon test and the reference frame method were used to analyze the association between microbiome abundance and disease phenotype.

Results: The relative abundance of the genus Corynebacterium was significantly higher in the pneumonia than in the non-pneumonia group. The microbiome analysis revealed significantly lower a-diversity in the NHAl group than in the non-NHAI group. In the analysis of $\beta$-diversity, the structure of the microbiome also differed significantly between the two groups (weighted UniFrac distance, Adonis, $P<0.001$ ). The abundance of Corynebacterium was significantly higher, and the relative abundances of Granulicatella, Staphylococcus, Streptococcus and Veillonella were significantly lower, in the NHAI group than in the nonNHAl group.

Conclusions: The microbiota signature of the ETAs distinguished between patients with and without risk factors for NHAI. The lung microbiome may serve as a therapeutic target for NHAl group.

\section{Background}

Among the recommendations of the 2016 and 2019 American Thoracic Society/Infectious Diseases Society of America (ATS/IDSA) guidelines was the removal of the category of healthcare-associated pneumonia (HCAP) [1, 2]. This is in contrast to the 2005 guidelines, which advocated the use of broadspectrum antibiotic therapy in HCAP and nosocomial pneumonia[3]. The change was based on several studies showing that, while patient characteristics such as comorbidities and age are determinants of the risk of multidrug-resistant (MDR) infection, this is not the case for all of the HCAP criteria [4-6]. Jones et al. showed that acceptance of the HCAP criteria lowered the threshold for broad-spectrum antibiotics for infection with nosocomial pathogens [7]. However, some HCAP criteria, such as admission from a nursing home or recent (preceding 90 days) hospitalization, are independent predictors of MDR infection [5, 8, 9]. For example, the risk of MDR infections in patients with HCAP requiring mechanical ventilation is similarly high to that of nosocomial infections $[10,11]$.

Based on previous studies, three risk factors may be better than the previous HCAP criteria for predicting the risk of infection with nosocomial pathogens: recent hospitalization, nursing home residence and antibiotic 
use in the past 90 days $[5,8,12]$. The application of new, culture-independent methods of characterizing microbial communities, such as $16 \mathrm{~S}$ rRNA, may lead to better characterization of patient risk groups and more appropriate therapeutic strategies[13]. We hypothesized that the composition of the bacterial microbiome in the respiratory tract during mechanical ventilation would be related to the newly defined risk factors for nursing-home and hospital-associated infections (NHAl), and to the 28-day all-cause and final hospital mortality rates. Therefore, we analyzed the microbial profiles of the endotracheal tube aspirates (ETAs) of intubated patients and determined the relationship between the ETA microbiome and risk factors for NCAI.

\section{Methods}

\section{Patients and study design}

Patients admitted to the intensive care unit (ICU) of Chuncheon Sacred Hospital (South Korea) between July 2017 and October 2018 were prospectively recruited. All patients placed on mechanical ventilation at the time of ICU admission were included in the study. The exclusion criteria were age $<18$ years, initiation of mechanical ventilation $>48 \mathrm{~h}$ after ICU admission and a duration of mechanical ventilation $<7$ days.

In 2005, the ATS/IDSA guidelines introduced HCAP as a new category of pneumonia requiring therapy similar to that prescribed for nosocomial pneumonia, namely broad-spectrum antibiotics[3]. However, several studies showed that HCAP is a heterogeneous disease, and that broad-spectrum antibiotic therapy may not be necessary for all patients with $\operatorname{HCAP}[6,8,14]$. Therefore, attempts were made to refine the definition of HCAP based on risk factors for MDR infections. On the basis of the available data[5, 8, 10, 12 , $14,15]$, we redefined NHAI patients as those meeting at least one of the following criteria: (1) nursing home resident with poor functional status; (2) recent (past 90 days) hospitalization; or (3) recent (past 180 days) antibiotic therapy.

The 60 patients ( 41 with and 19 without pneumonia) enrolled in this study were divided into NHAI and nonNHAl groups and prospectively followed. This study was approved by the Institutional Review Board of Chuncheon Sacred Heart Hospital (IRB approval number: 2017-47).

\section{Data collection and clinical outcome measures}

Data on demographic characteristics (age, sex, preexisting comorbidities), the indications for intubation, the presence of acute respiratory distress syndrome (ARDS), the arterial oxygen tension/fraction of inspired oxygen (SaO2/FiO2) ratio, and the Glasgow Coma Scale (GCS), Acute Physiology and Chronic Health Evaluation (APACHE) II) and Sequential Organ Failure Assessment (SOFA) scores were recorded. The Charlson Comorbidity Index (CCl) score was calculated as described previously[16]. The clinical outcomes of interest in this study were the 28-day all-cause and final hospital mortality rates. Respiratory samples were acquired by endotracheal aspiration, with serial samples collected 1, 3 and 7 days after the initiation of mechanical ventilation.

\section{DNA extraction, PCR and sequencing}


Genomic DNA was extracted using a commercial microbial DNA isolation kit (MP Biomedicals, USA) and then amplified using primers targeting the V3-V4 region of the prokaryotic 16S rRNA gene. The amplicons were purified using XSEP MagBead (CELEMICS), and then subjected to PCR using the Nextera Index Kit (Illumina, USA) following the manufacturer's instructions. The resultant product was further purified using XSEP MagBead (CELEMICS). The prepared bacterial amplicon library was then quantified, mixed with multiple libraries and sequenced using the MiSeq v3 platform (Illumina).

The raw sequencing data files were preprocessed before downstream data analysis. To remove sequences with low-quality scores, the raw sequence reads were pre-filtered using PRINSEQ[17]. Adapter sequences were removed using CUTADAPT[18]. Paired-end reads were merged using PEAR[19] and then filtered with PRINSEQ. Chimeric sequences and singletons were screened and reduced using USEARCH[20]. Finally, 180 samples were analyzed and an average of 133,219 reads per sample were obtained (minimum, 29,247; maximum, 587,337). The downstream data analysis was carried out using QIIME[21] with the EzBioCloud 16S rRNA gene sequence database[22]. Operational taxonomic units (OTUs) were defined as clusters of sequences with $\geq 97 \%$ identity.

\section{Data processing and statistical analysis}

Various Microbial community comparisons were performed using Quantitative Insights in Microbial Ecology software (QIIME, v. 1.8) and the R package Vegan.

The microbial sequences from the ETAs represented 554 genera and 2,009 species. Each subject was sequenced twice at two different time points (day 1 and day 3 or 7). Absolute OTU of each subject for both time points was combined, and species were filtered by two criteria; 1 ) eliminating strains that appear only in a few patients, 2) removing strains that account for less than 1000 OTU. Finally, 11 predominant genera (Acinetobacter, Streptococcus, Corynebacterium, Staphylococcus, Prevotella, Neisseria, Veillonella, Mycoplasma, Granulicatella, Actinomyces, Campylobacter) and 8 predominant species (Acinetobacter baumannii, Streptococcus mitis, Corynebacterium ulcerans, Staphylococcus caprae, Veillonell adispar, Granulicatella adiacens, Streptococcus parasanguinis, Streptococcus lactarius) were included in the statistical analysis.

Ace, Chao1, the Shannon index and the Simpson index were used to express a-diversity; $\beta$-diversity (intersample diversity) was defined as the extent of the similarity between microbial communities based on the degree of structural overlap. Nonmetric multidimensional scaling plots based on weighted UniFrac distances were used to visualize the differences between groups in microbial community structure. Principle coordinate analysis was conducted using a permutational multivariate ANOVA (PERMANOVA), performed via the Adonis function of the $\mathrm{R}$ package vegan (1,000 permutations).

We conducted statistical analysis to identify the association of pneumonia and NHAI risk factor on the abundance with each OTU. Each subject was measured at two different time points and generalized linear mixed effects models (GLMM) was utilized to handle the repeatedly observed measurement. Pneumonia and NHAI risk factor were considered as response variables. Relative abundances of OTUs at each time point were Log2 transformed, and its effect was evaluated by including it as a covariate. We also 
considered the paired and longitudinal distance-based approach (Pldist) to detect the longitudinal changes in the microbiome over time with various outcome types[23]. It can also calculate differences in nonphylogenetic dissimilarities such as Gower's distance, Bray-Curtis dissimilarities, Jaccard distance and Kulczynski distance between time points. Last we found that pneumonia and NHAl risk factor are timeinvariant and thus considered three methods for cross-sectional data; phylogenetic tree-based microbiome association test (TMAT), Wilcoxon test and reference frame method[24, 25]. For the cross-sectional methods, means of log-transformed read count per million (CPM) at two different point were considered as the response variables. Among all approaches, only GLMM and TMAT can adjust the effect of the other covariates and age, sex, APACHE score and CCI were included as covariates for both. GLMM was conducted with SAS 9.4 (Cary, NC), and the other analyses were done with R software. All test results were considered significant using a $p$ value of $\leq 0.05$.

\section{Results}

\section{Characteristics of the study participants}

The study population consisted of 60 patients who had been placed on mechanical ventilation, including 41 patients diagnosed with pneumonia and 24 who had risk factors for NHAl. Table 1 shows the patient characteristics. The proportions of ARDS, a high C-reactive protein (CRP) level and an abnormal paO2/FiO2 ratio were higher in patients with than without pneumonia, as expected. Patients in the NHAI and non-NHAI groups did not differ in terms of sex, severity index (APACHE and SOFA) scores, or the GCS score. However, patients in the NHAl group were older and had more comorbidities than the non-NHAl group. There were no significant differences between the two groups in either the 28-day all-cause or final hospital mortality rate. 
Table 1

Baseline characteristics of the study participants.

\begin{tabular}{|c|c|c|c|c|c|c|}
\hline & Pneumonia & $\begin{array}{l}\text { Non- } \\
\text { pneumonia }\end{array}$ & $\begin{array}{l}P \\
\text { value }\end{array}$ & NHAI & Non NHAl & $\begin{array}{l}P \\
\text { value }\end{array}$ \\
\hline & $n=41$ & $n=19$ & & $n=24$ & $n=36$ & \\
\hline Age 9 & $73(61-79)$ & $76(59-81)$ & 0.744 & $78(72-85)$ & $71(57-77)$ & 0.004 \\
\hline Male $^{\dagger}$ & $30(73.2 \%)$ & $12(63.2 \%)$ & 0.431 & $15(62.5 \%)$ & $27(75.0 \%)$ & 0.391 \\
\hline ARDS & $8(19.5 \%)$ & $0(0 \%)$ & 0.047 & $4(16.7 \%)$ & $4(11.1 \%)$ & 0.702 \\
\hline Pneumonia & & & & $23(95.8 \%)$ & $19(52.8 \%)$ & $\begin{array}{l}< \\
0.001\end{array}$ \\
\hline $\begin{array}{l}\text { Charlson Comorbidity } \\
\text { Index }\end{array}$ & $3(1-4)$ & & 0.085 & $3(2.3-5.0)$ & $1.5(0-2.8)$ & $\begin{array}{l}< \\
0.001\end{array}$ \\
\hline Cause of intubation & & & $<001$ & & & $\begin{array}{l}< \\
0.001\end{array}$ \\
\hline Cardiac arrest & $1(2.4 \%)$ & $3(15.8 \%)$ & & $0(0.0 \%)$ & $4(11.1 \%)$ & \\
\hline Neurological distress & $5(12.2 \%)$ & $13(68.4 \%)$ & & $1(4.2 \%)$ & $17(47.2 \%)$ & \\
\hline Post-operative status & $0(0 \%)$ & $1(5.3 \%)$ & & $0(0.0 \%)$ & $1(2.8 \%)$ & \\
\hline Respiratory & $35(85.4 \%)$ & $2(10.5 \%)$ & & $23(95.8 \%)$ & $14(38.9 \%)$ & \\
\hline $\mathrm{PaO} 2 / \mathrm{FiO} 2$ ratio & $\begin{array}{l}212 \\
(133.5- \\
299)\end{array}$ & $\begin{array}{l}431(321- \\
458)\end{array}$ & $<.001$ & $\begin{array}{l}222(131.3- \\
301.0)\end{array}$ & $\begin{array}{l}323(184.3- \\
442.5)\end{array}$ & 0.022 \\
\hline Severity & & & & & & \\
\hline APACHE II score & $20(16-24)$ & $22(17-25)$ & 0.202 & $\begin{array}{l}20.5(16.3- \\
24.0)\end{array}$ & $21(17-25)$ & 0.634 \\
\hline SOFA score & $7(6-9)$ & $6(4-9)$ & 0.117 & $7(6-9)$ & $7(5-9)$ & 0.569 \\
\hline $\mathrm{GCS}^{9}$ & $8(6-11)$ & $6(5-9)$ & 0.074 & $8.5(6.0-10.8)$ & $7.5(6-9)$ & 0.303 \\
\hline $\begin{array}{l}\text { Extubation success in } \\
3 \text { weeks }\end{array}$ & $23(56.1 \%)$ & $11(57.9 \%)$ & 0.999 & $14(58.3 \%)$ & $20(55.6 \%)$ & 0.999 \\
\hline $\begin{array}{l}\text { 28-day all-cause } \\
\text { mortality }\end{array}$ & $12(29.3 \%)$ & $6(31.6 \%)$ & 0.999 & $7(29.2 \%)$ & $11(30.6 \%)$ & 0.999 \\
\hline $\begin{array}{l}\text { Final hospital } \\
\text { mortality }\end{array}$ & $19(46.3 \%)$ & $6(31.7 \%)$ & 0.4 & $11(45.8 \%)$ & $14(38.9 \%)$ & 0.606 \\
\hline MV duration & $13(8-18)$ & $10(7-16)$ & 0.335 & $13(8-17.5)$ & $\begin{array}{l}10(7.0- \\
16.8)\end{array}$ & 0.384 \\
\hline
\end{tabular}

ฯ median (interquartile range) tfrequency (\%) § median (range) 


\begin{tabular}{|c|c|c|c|c|c|c|}
\hline & Pneumonia & $\begin{array}{l}\text { Non- } \\
\text { pneumonia }\end{array}$ & $\begin{array}{l}P \\
\text { value }\end{array}$ & NHAl & Non NHAI & $\begin{array}{l}P \\
\text { value }\end{array}$ \\
\hline & $\mathrm{n}=41$ & $n=19$ & & $\mathrm{n}=\mathbf{2 4}$ & $n=36$ & \\
\hline $\mathrm{CRP}(\mathrm{mg} / \mathrm{dL})$ & $\begin{array}{l}133(45- \\
213.5)\end{array}$ & $21(5-132)$ & 0.008 & $\begin{array}{l}124.5(62.8- \\
192.0)\end{array}$ & $\begin{array}{l}94(8.0- \\
194.0)\end{array}$ & 0.127 \\
\hline
\end{tabular}

Differences in the microbial composition of the ETAs between patient groups (pneumonia vs nonpneumonia, NHAI vs. non-NHAI) are shown in the taxonomic summary (Fig. 1). The average abundance of the genera Corynebacterium, Staphylococcus and Pseudomonas was higher in the pneumonia group than in the non-pneumonia group. The relative abundances of Streptococcus and Prevotella were higher in the non-NHAl group, whereas the relative abundance of Corynebacterium was higher in the NHAI group.

\section{Respiratory microbiota during mechanical ventilation in patients with and without pneumonia}

Neither a-diversity nor $\beta$-diversity differed significantly between patients with and without pneumonia. The association between pneumonia and microbial taxa was analyzed (Table 2), adjusting for the effects of age, sex, and APACHE and CCI scores in the TMAT, optimal microbiome-based association test (OMiAT), and GLMM, but not in the Wilcoxon test. A correlation between Corynebacterium and pneumonia was demonstrated by the TMAT, while the GLMMs identified a significant difference in the abundance of $C$. ulcerans between patients with and without pneumonia $(P=0.035)$. 
Table 2

Bacterial genera associated with pneumonia.

NHAl: nursing-home- and hospitalassociated infections

ARDS: acute respiratory distress syndrome

\section{APACHE II: Acute Physiology and} Chronic Health Evaluation II

SOFA: Sequential Organ

Failure Assessment

GCS: Glasgow

Coma Scale

MV: mechanical

ventilation

CRP: C-reactive

protein

\begin{tabular}{|c|c|c|c|c|c|c|c|c|}
\hline \multirow[t]{2}{*}{ Genus } & FDR- & Beta & FDR- & Estimate- & \multirow{2}{*}{$\begin{array}{l}\text { FDR- } \\
\text { RF }\end{array}$} & FDR- & $\beta-$ & FDR- \\
\hline & OMiAT & TMAT & TMAT & RF & & Wilcoxon & GLMM & GLMM \\
\hline$A c$ & 0.7832 & 0.02 & 0.7321 & NA & NA & 0.6809 & 0.014 & 0.7855 \\
\hline
\end{tabular}

FDR: false discovery rate

OMiAT: optimal microbiome-based association test

TMAT: phylogenetic-tree-based microbiome association test

RF: reference frame

GLMM: generalized linear mixed model 


\section{NHAl: nursing-home and hospital- associated infections}

\section{ARDS: acute respiratory distress syndrome}

\section{APACHE II: Acute Physiology and Chronic Health Evaluation II}

\begin{tabular}{|c|c|c|c|c|c|c|c|c|}
\hline Corynebacterium & 0.2791 & 0.1 & 0.0226 & -2.03 & 0.9985 & 0.005 & 0.102 & 0.4899 \\
\hline Granulicatella & 0.7832 & -0.04 & 0.3564 & -1.89 & 0.9985 & 0.3817 & -0.104 & 0.4899 \\
\hline Staphylococcus & 0.7832 & $\overline{0} .01$ & 0.8202 & 0.09 & 0.9985 & 0.9556 & 0.026 & 0.725 \\
\hline Streptococcus & 0.2791 & $\overline{0} .03$ & 0.7321 & 0 & 0.9985 & 0.3817 & -0.04 & 0.6638 \\
\hline Veillonella & 0.7832 & $\overline{0} .03$ & 0.708 & 0.35 & 0.9985 & 0.6809 & $\overline{0} .065$ & 0.5494 \\
\hline
\end{tabular}

FDR: false discovery rate

OMiAT: optimal microbiome-based association test

TMAT: phylogenetic-tree-based microbiome association test

RF: reference frame

GLMM: generalized linear mixed model

Figure 2a shows the increased relative abundance of the genus Corynebacterium in the pneumonia than in the non-pneumonia group. The difference of this genus abundance was mainly caused by a species named C. ulcerans. No other Corynebacterium species detected in the samples showed meaningful difference between the pneumonia and non-pneumonia groups. Figure $2 \mathrm{~b}$ shows the larger increase in $C$. ulcerans in the pneumonia than in the non-pneumonia group, both on day 1 and on day 7. A trend towards a larger difference in the OTU abundance of Corynebacterium in the pneumonia group compared to the nonpneumonia group on day 7 was also identified.

\section{Respiratory microbiota during mechanical ventilation in patients with and without risk factors for NHAI}

An analysis of a-diversity revealed significant differences between the NHAl and non-NHAl groups (expressed as median [interquartile range]; Fig. 3a). Ace $(67.5[48.2,108.9]$ vs. $139.9[106.2,215.7] ; P<$ $0.001)$, Chao $(64.4[45.3,112.0]$ vs. $137.5[106.2,230.6] ; P<0.001)$, Shannon index $(2.3[0.7,2.9]$ vs. 3.8 $[2.3,4.7] ; P<0.001)$ and Simpson index (0.7 [0.2,0.8] vs. $0.9[0.6,0.9] ; P<0.001)$ values were lower in the NHAl group than in the non-NHAI group. The microbiome structure of the NHAI group could also be clearly distinguished from that of the non-NHAl group based on the $\beta$-diversity (PERMANOVA, adjusted $P<0.001$, Fig. 3b). 
The microbial taxa associated with NHAI were identified using the methods shown in Table 3. Adjustments were made for age, sex, and the APACHE and CCI scores in the TMAT, OMIAT, and GLMMs, but not in the Wilcoxon test. The TMAT and Wilcoxon analyses revealed that Granulicatella, Streptococcus and Veillonella were negatively correlated with NHAl, while Corynebacterium was positively correlated (Table 3).

Table 3

Bacterial genera associated with NHAl.

\begin{tabular}{|c|c|c|c|c|c|c|c|c|}
\hline Genus & $\begin{array}{l}\text { FDR- } \\
\text { OMiAT }\end{array}$ & $\begin{array}{l}\beta- \\
\text { TMAT }\end{array}$ & $\begin{array}{l}\text { FDR- } \\
\text { TMAT }\end{array}$ & $\begin{array}{l}\text { Estimate- } \\
\text { RF }\end{array}$ & $\begin{array}{l}\text { FDR- } \\
\text { RF }\end{array}$ & $\begin{array}{l}\text { FDR- } \\
\text { Wilcoxon }\end{array}$ & $\begin{array}{l}\text { Beta- } \\
\text { GLMM }\end{array}$ & $\begin{array}{l}\text { FDR- } \\
\text { GLMM }\end{array}$ \\
\hline Acinetobacter & 0.5861 & -0.08 & 0.0807 & & NA & 0.52 & -0.039 & 0.6165 \\
\hline Corynebacterium & 0.1788 & 0.2 & 0.0004 & 3.21 & 0.3302 & $<0.05$ & 0.17 & 0.2166 \\
\hline Granulicatella & 0.1911 & -0.18 & 0.0002 & -1.15 & 0.8524 & $<0.05$ & -0.318 & 0.0385 \\
\hline Staphylococcus & 0.2129 & -0.14 & 0.023 & -2.22 & 0.8524 & 0.344 & -0.142 & 0.2166 \\
\hline Streptococcus & 0.2129 & -0.09 & 0.0132 & 3.1 & 0.8524 & 0.0045 & -0.067 & 0.4439 \\
\hline Veillonella & 0.5861 & -0.11 & 0.0101 & -0.39 & 0.8611 & 0.0045 & -0.136 & 0.2166 \\
\hline \multicolumn{9}{|c|}{ FDR: false discovery rate } \\
\hline \multicolumn{9}{|c|}{ OMiAT: optimal microbiome-based association test } \\
\hline \multicolumn{9}{|c|}{ TMAT: phylogenetic-tree-based microbiome association test } \\
\hline \multicolumn{9}{|c|}{ RF: reference frame } \\
\hline \multicolumn{9}{|c|}{ GLMM: generalized linear mixed model } \\
\hline
\end{tabular}

Similarly, the GLMMs revealed that the genera Granulicatella and Prevotella were more abundant in the nonNHAl group, while at the species level $C$. ulcerans was more abundant in the NHAl group and Granulicatella adiacens was more abundant in the non-NHAl group (Supplementary Table 1).

Supplementary Fig. 1 shows the longitudinal trends in microbial taxa according to the risk factors for NHAI. In ETAs from days 1 and 7, the OTU abundances of Veillonella, Granulicatella and Streptococcus were significantly higher in the non-NHAl than in the NHAl group, whereas Corynebacterium was more abundant in the NHAl group. However, none of the longitudinal changes in microbial taxa differed significantly by disease phenotype.

\section{Longitudinal change in the respiratory microbiome according to clinical outcome}

The associations between non-phylogenetic dissimilarities (based on pldist) and the clinical outcomes (the 28-day all-cause and final hospital mortality rates) were analyzed using the microbiome regression-based kernel association test (MiRKAT). The results showed that the variability in the respiratory microbiome was not associated with the clinical outcome (Table 4). The GLMM analysis yielded similar results. There was 
no association of microbial OTU abundance with the 28-day all-cause or final hospital mortality rate, in either the pneumonia or non-pneumonia group (Supplementary Table 2).

Table 4

Association between microbiome variability and clinical outcome. $P$ values are from the MiRKAT. The quantitative and qualitative analyses (Bray-Curtis, Jaccard, Kulczynski and Gower analyses) were adjusted for sex, age, and APACHE II and CCl scores.

\begin{tabular}{|c|c|c|c|c|}
\hline & \multicolumn{2}{|c|}{ 28-day all-cause mortality } & \multicolumn{2}{|c|}{ Final hospital mortality } \\
\hline & Species & Genus & Species & Genus \\
\hline \multicolumn{5}{|c|}{ Total $(n=60)$} \\
\hline Bray-Curtis & 0.15 & 0.63 & 0.45 & 0.24 \\
\hline Jaccard & 0.33 & 0.57 & 0.48 & 0.22 \\
\hline Kulczynski & 0.34 & 0.58 & 0.42 & 0.32 \\
\hline Gower & 0.29 & 0.45 & 0.43 & 0.23 \\
\hline \multicolumn{5}{|c|}{ Pneumonia $(n=41)$} \\
\hline Bray-Curtis & 0.3 & 0.76 & 0.54 & 0.36 \\
\hline Jaccard & 0.51 & 0.63 & 0.43 & 0.26 \\
\hline Kulczynski & 0.5 & 0.69 & 0.28 & 0.3 \\
\hline Gower & 0.29 & 0.63 & 0.38 & 0.44 \\
\hline \multicolumn{5}{|c|}{ Non-pneumonia $(n=19)$} \\
\hline Bray-Curtis & 0.65 & 0.14 & 0.65 & 0.14 \\
\hline Jaccard & 0.66 & 0.5 & 0.66 & 0.5 \\
\hline Kulczynski & 0.75 & 0.62 & 0.75 & 0.62 \\
\hline Gower & 0.71 & 0.13 & 0.71 & 0.13 \\
\hline
\end{tabular}

\section{Discussion}

Patients with HCAP are a heterogeneous group[14]. Because the existing HCAP criteria did not reflect the risk of nosocomial pathogen infection and led to overuse of broad-spectrum antibiotics, they were omitted from the 2016 hospital-acquired pneumonia/ventilator-associated pneumonia (HAP/VAP) guidelines [1, 7]. Attempts to develop alternative criteria for HCAP, and a specific therapy $[8,11,14]$, included our concept of $\mathrm{NHAl}$, which may better predict the occurrence of MDR infections and consequences of continued exposure to hospital and other healthcare environments. 
Recent studies using conventional microbiologic culture tests have shown different patterns of infection among countries and geographic regions [26-29]. Studies from the USA and the Asia/Pacific region reported that HCAP is characterized by high frequencies of infections with drug-resistant pathogens,[26-28, 30] whereas other studies showed similarities in the involved pathogens between HCAP and communityacquired pneumonia (CAP).[29]

Culture-independent 16S rRNA gene sequencing is useful for detecting fastidious bacteria, the abundance of which tends to be underestimated; this method is more sensitive than conventional culture methods [31]. To our knowledge, this is the first study to use microbiome sequencing to determine the associations of healthcare/hospital-related factors with the composition of the respiratory microbiome of intubated patients.

The most important finding of this study was that the ETA microbiome profile of intubated patients was significantly associated with newly defined risk factors for NHAl. Generally, microbial diversity was higher in patients without than with risk factors for NHAl. The analysis of $\beta$-diversity, based on weighted UniFrac distances, showed distinct clusters of samples, identified as NHAI and non-NHAI groups. Beyond microbial diversity, our study showed the association of specific bacterial genera with continued exposure to healthcare and hospital environments.

The abundance of Corynebacterium increased, whereas the abundances of Granulicatella, Streptococcus, Staphylococcus and Veillonella decreased, in intubated patients with risk factors for NHAI. In particular, members of the genus Corynebacterium were significantly more abundant in intubated patients with than without pneumonia, and the abundance of $C$. ulcerans in the pneumonia group tended to increase over time. Although Corynebacterium spp. are among the commensal flora of the nasopharynx[32], a causative role in pneumonia was recently described[33-36]. The identification of Corynebacteria subspecies is now possible using new diagnostic technologies, such as matrix-assisted laser desorption ionization time of flight mass spectrometry and $16 \mathrm{~S}$ rRNA gene sequencing[13, 33-35, 37]. Based on such studies, Clariot et al. suggested that Corynebacterium spp. may be responsible for pneumonia in mechanically ventilated patients, similar to our results[33], while Yasuda et al. reported that $C$. ulcerans produces diphtheria toxin and causes severe pneumonia complicated by diffuse pseudomembrane formation in the central airways[36]. Corynebacterium spp. may also be responsible for HAP. An analysis of bronchoalveolar lavage fluid (BALF) obtained from Japanese patients revealed Corynebacterium spp. in the samples of $11.8 \%$ of the patients with HAP, and in $4.9 \%$ of those from patients with HCAP; however, Corynebacterium spp. was present in only $1.6 \%$ of the samples from patients with CAP[37]. Our results also demonstrate the need to consider Corynebacterium spp. as a possible healthcare/hospital-associated pathogen.

Several studies using molecular techniques have revealed the presence of unexpected microorganisms in specific phenotypes of pneumonia. For example, Steenhuisen et al. found an increase in Lactobacilus and Rothia in CAP, while Zarkharkina et al. reported a decrease in these microorganisms, in patients with VAP $[38,39]$. Similar to our own findings, a study of adult ICU patients identified Prevotella as being dominant in controls, whereas Corynebacteria species were uniquely present in VAP cohorts [40]. 
Segal et al. reported that a lung microbiome containing taxa from the oral cavity (i.e., Streptococcus, Veillonella, Granulicatella, and Prevotella) induced host cellular mucosal immunity of the Th17/neutrophilic phenotype and blunted TLR4 responses [41]. In the study of Kitsios et al., the dominance of oral taxa within lung communities was strongly associated with culture negativity and weaker host immune responses [42]. From these data, it can be inferred that non-NHAl patients in whom oral taxa dominate the lung microbiome will have a weaker immune response than NHAl patients, which would also explain why patients with HCAP present with more severe disease and have clinical outcomes resembling those of nosocomial pneumonia $[26,28,43]$.

Our data showed no difference in microbial community according to clinical outcome; this is in contrast to Dickson et al., who reported that the lung microbiome predicts outcomes in critically ill patients [44]. This discordance may be due to differences in study population and sampling method (ETA vs. BALF).

Our study had several limitations. First, it used a single-center design and the sample size was small. Multicenter studies with larger populations are needed to validate our results. Second, we did not obtain BALF samples, although this was in accordance with ATS guidelines recommending non-invasive testing[1]. Nevertheless, analyzing BAL and ETA samples may enable comparison with the results of other studies, such that more robust conclusions could be drawn. Third, although our findings support the concept of an $\mathrm{NHAl}$ risk factor-associated microbiome, its clinical significance should be investigated via functional analyses, for example. Such studies would allow conclusions to be drawn regarding the association between an NHAl-specific microbiome and outcome/immune metabolic responses.

The strengths of our study included the homogeneity of the study population in terms of age (all elderly patients), and the multiple serial samples acquired from each patient. The analysis showed that the composition of the NHAl-related microbiome was similar regardless of antibiotic use or time of day.

\section{Conclusions}

In this prospective observational cohort study of mechanically ventilated patients, the loss of diversity and dysbiosis of the respiratory microbiome were more profound in patients with than without risk factors for $\mathrm{NHAl}$, which were in turn positively associated with the presence of Corynebacterium, and negatively associated with that of Granulicatella, Streptococcus, Staphylococcus and Veillonella. Prospective studies are needed to determine the clinical relevance of microbial community profiling, and to identify novel therapeutic targets in critically ill elderly patients with risk factors for NHAI.

\section{Additional information}

The supplementary figures and tables can be found in the Supplemental Materials section of the online article.

\section{List Of Abbreviations}

HCAP: Healthcare-associated pneumonia 
NHAl: nursing home and hospital associated infection

ICU: intensive care unit

TMAT: tree-based microbiome association test

GLMM: generalized linear mixed effects models

ETA: endotracheal tube aspirates

OTU: Operational taxonomic units

CAP: community acquired pneumonia

VAP: ventilator associated pneumonia

MDR: multidrug resistant

APACHE II: Acute Physiology, Age, Chronic Health Evaluation II

SOFA: Sequential Organ Failure Assessment

CCl: Charlson comorbidity index

\section{Declarations}

\section{Ethics approval and consent to participate}

This study was approved by the Institutional Review Board of Chuncheon Sacred Heart Hospital (IRB approval number: 2017-47). Informed consent was obtained from all patients or their legal representatives. This research was conducted in accordance with the Declaration of Helsinki.

\section{Consent for publication}

Not applicable.

\section{Availability of data and materials}

The datasets used and/or analysed during the current study are available from the corresponding author on reasonable request.

\section{Competing interests}

The authors declare that they have no competing interests.

\section{Funding}


This study was supported by the Bio \& Medical Technology Development Program of the National Research Foundation (NRF) funded by the Korean government (MSIT) (NRF-2017M3A9E8033225) and by the National Research Foundation of Korea Grant funded by Korean Government (NRF 2020R1A2C1011455). This research was supported by Hallym University Research Fund. Funding agencies had no role in the study design, data collection and analysis, decision to publish, manuscript preparation, or decision to submit the manuscript for publication. The remaining authors have no conflicts of interest to report.

Authors' Contributions:

MB, CB, SW, YK and HY had the idea for and designed the study and had full access to all data in the study and take responsibility for the integrity of the data analysis. JYH and JJL wrote the first full draft of the report. MB, NEK, CB, SW, YK, JJL, HY and JYH contributed to critical revision of the report. NEK, SW and HY contributed to the statistical analysis. All authors contributed to data acquisition, data analysis, or data interpretaion, and reviewed and approved the final version.

\section{Acknowledgements}

The authors thank all of study participants and the medical staff from Chuncheon Sacred Heart Hospital for their contributions to this study.

\section{References}

1. Kalil AC, Metersky ML, Klompas M, Muscedere J, Sweeney DA, Palmer LB, Napolitano LM, O'Grady NP, Bartlett JG, Carratala J, et al: Management of Adults With Hospital-acquired and Ventilator-associated Pneumonia: 2016 Clinical Practice Guidelines by the Infectious Diseases Society of America and the American Thoracic Society. Clin Infect Dis 2016, 63:e61-e111.

2. Metlay JP, Waterer GW, Long AC, Anzueto A, Brozek J, Crothers K, Cooley LA, Dean NC, Fine MJ, Flanders SA, et al: Diagnosis and Treatment of Adults with Community-acquired Pneumonia. An Official Clinical Practice Guideline of the American Thoracic Society and Infectious Diseases Society of America. Am J Respir Crit Care Med 2019, 200:e45-e67.

3. American Thoracic S, Infectious Diseases Society of A: Guidelines for the management of adults with hospital-acquired, ventilator-associated, and healthcare-associated pneumonia. Am J Respir Crit Care Med 2005, 171:388-416.

4. Chalmers JD, Rother C, Salih W, Ewig S: Healthcare-associated pneumonia does not accurately identify potentially resistant pathogens: a systematic review and meta-analysis. Clin Infect Dis 2014, 58:330339.

5. Gross AE, Van Schooneveld TC, Olsen KM, Rupp ME, Bui TH, Forsung E, Kalil AC: Epidemiology and predictors of multidrug-resistant community-acquired and health care-associated pneumonia. Antimicrob Agents Chemother 2014, 58:5262-5268.

6. Yap V, Datta D, Metersky ML: Is the Present Definition of Health Care-Associated Pneumonia the Best Way to Define Risk of Infection with Antibiotic-Resistant Pathogens? Infectious Disease Clinics of 
North America 2013, 27:1-+.

7. Jones BE, Jones MM, Huttner B, Stoddard G, Brown KA, Stevens VW, Greene T, Sauer B, Madaras-Kelly $\mathrm{K}$, Rubin M, et al: Trends in Antibiotic Use and Nosocomial Pathogens in Hospitalized Veterans With Pneumonia at 128 Medical Centers, 2006-2010. Clin Infect Dis 2015, 61:1403-1410.

8. Park SC, Kang YA, Park BH, Kim EY, Park MS, Kim YS, Kim SK, Chang J, Jung JY: Poor prediction of potentially drug-resistant pathogens using current criteria of health care-associated pneumonia. Respir Med 2012, 106:1311-1319.

9. Schreiber MP, Chan CM, Shorr AF: Resistant pathogens in nonnosocomial pneumonia and respiratory failure: is it time to refine the definition of health-care-associated pneumonia? Chest 2010, 137:12831288.

10. El-Solh AA, Sikka P, Ramadan F, Davies J: Etiology of severe pneumonia in the very elderly. Am J Respir Crit Care Med 2001, 163:645-651.

11. El Solh AA, Pietrantoni C, Bhat A, Bhora M, Berbary E: Indicators of potentially drug-resistant bacteria in severe nursing home-acquired pneumonia. Clin Infect Dis 2004, 39:474-480.

12. Shorr AF, Zilberberg MD, Micek ST, Kollef MH: Prediction of infection due to antibiotic-resistant bacteria by select risk factors for health care-associated pneumonia. Arch Intern Med 2008, 168:2205-2210.

13. Xia LP, Bian LY, Xu M, Liu Y, Tang AL, Ye WQ: 16S rRNA gene sequencing is a non-culture method of defining the specific bacterial etiology of ventilator-associated pneumonia. Int J Clin Exp Med 2015, 8:18560-18570.

14. Brito V, Niederman MS: Healthcare-associated pneumonia is a heterogeneous disease, and all patients do not need the same broad-spectrum antibiotic therapy as complex nosocomial pneumonia. Curr Opin Infect Dis 2009, 22:316-325.

15. Umeki K, Tokimatsu I, Yasuda C, Iwata A, Yoshioka D, Ishii H, Shirai R, Kishi K, Hiramatsu K, Matsumoto $B$, Kadota J: Clinical features of healthcare-associated pneumonia (HCAP) in a Japanese community hospital: comparisons among nursing home-acquired pneumonia (NHAP), HCAP other than NHAP, and community-acquired pneumonia. Respirology 2011, 16:856-861.

16. Charlson ME, Pompei P, Ales KL, MacKenzie CR: A new method of classifying prognostic comorbidity in longitudinal studies: development and validation. J Chronic Dis 1987, 40:373-383.

17. Schmieder R, Edwards R: Quality control and preprocessing of metagenomic datasets. Bioinformatics 2011, 27:863-864.

18. Martin M: Cutadapt removes adapter sequences from high-throughput sequencing reads. EMBnryjournal 2011, 17.

19. Zhang J, Kobert K, Flouri T, Stamatakis A: PEAR: a fast and accurate Illumina Paired-End reAd mergeR. Bioinformatics 2014, 30:614-620.

20. Edgar RC: Search and clustering orders of magnitude faster than BLAST. Bioinformatics 2010, 26:2460-2461.

21. Caporaso JG, Kuczynski J, Stombaugh J, Bittinger K, Bushman FD, Costello EK, Fierer N, Pena AG, Goodrich JK, Gordon JI, et al: QIIME allows analysis of high-throughput community sequencing data. 
Nat Methods 2010, 7:335-336.

22. Yoon SH, Ha SM, Kwon S, Lim J, Kim Y, Seo H, Chun J: Introducing EzBioCloud: a taxonomically united database of 16S rRNA gene sequences and whole-genome assemblies. Int J Syst Evol Microbiol 2017, 67:1613-1617.

23. Plantinga AM, Chen J, Jenq RR, Wu MC: pldist: ecological dissimilarities for paired and longitudinal microbiome association analysis. Bioinformatics 2019, 35:3567-3575.

24. Kim KJ, Park J, Park SC, Won S: Phylogenetic tree-based microbiome association test. Bioinformatics 2020, 36:1000-1006.

25. Morton JT, Marotz C, Washburne A, Silverman J, Zaramela LS, Edlund A, Zengler K, Knight R: Establishing microbial composition measurement standards with reference frames. Nat Commun 2019, 10:2719.

26. Kollef MH, Shorr A, Tabak YP, Gupta V, Liu LZ, Johannes RS: Epidemiology and outcomes of healthcare-associated pneumonia: results from a large US database of culture-positive pneumonia. Chest 2005, 128:3854-3862.

27. Micek ST, Kollef KE, Reichley RM, Roubinian N, Kollef MH: Health care-associated pneumonia and community-acquired pneumonia: a single-center experience. Antimicrob Agents Chemother 2007, 51:3568-3573.

28. Shindo Y, Sato S, Maruyama E, Ohashi T, Ogawa M, Hashimoto N, Imaizumi K, Sato T, Hasegawa Y: Health-care-associated pneumonia among hospitalized patients in a Japanese community hospital. Chest 2009, 135:633-640.

29. Chalmers JD, Taylor JK, Singanayagam A, Fleming GB, Akram AR, Mandal P, Choudhury G, Hill AT: Epidemiology, antibiotic therapy, and clinical outcomes in health care-associated pneumonia: a UK cohort study. Clin Infect Dis 2011, 53:107-113.

30. Park HK, Song JU, Um SW, Koh WJ, Suh GY, Chung MP, Kim H, Kwon OJ, Jeon K: Clinical characteristics of health care-associated pneumonia in a Korean teaching hospital. Respir Med 2010, 104:1729-1735.

31. Kelly BJ, Imai I, Bittinger K, Laughlin A, Fuchs BD, Bushman FD, Collman RG: Composition and dynamics of the respiratory tract microbiome in intubated patients. Microbiome 2016, 4:7.

32. Lipsky BA, Goldberger AC, Tompkins LS, Plorde JJ: Infections caused by nondiphtheria corynebacteria. Rev Infect Dis 1982, 4:1220-1235.

33. Clariot S, Constant O, Lepeule R, Fihman V, Razazi K, Cook F, Attias A, Merle JC, Hemery F, Levesque E, et al: Clinical relevance and impact of Corynebacterium isolation in lower respiratory tract of critically ill patients requiring mechanical ventilation. Infection 2020.

34. Yatera K, Mukae $\mathrm{H}$ : Corynebacterium species as one of the major causative pathogens of bacterial pneumonia. Respir Investig 2020.

35. Yang K, Kruse RL, Lin WV, Musher DM: Corynebacteria as a cause of pulmonary infection: a case series and literature review. Pneumonia (Nathan) 2018, 10:10.

36. Yasuda I, Matsuyama H, Ishifuji T, Yamashita Y, Takaki M, Morimoto K, Sekino M, Yanagihara K, Fujii T, Iwaki M, et al: Severe Pneumonia Caused by Toxigenic Corynebacterium ulcerans Infection, Japan. 
Emerg Infect Dis 2018, 24:588-591.

37. Yatera K, Noguchi S, Yamasaki K, Kawanami T, Fukuda K, Naito K, Akata K, Kido T, Ishimoto H, Sakamoto N, et al: Determining the Possible Etiology of Hospital-Acquired Pneumonia Using a Clone Library Analysis in Japan. Tohoku J Exp Med 2017, 242:9-17.

38. de Steenhuijsen Piters WA, Huijskens EG, Wyllie AL, Biesbroek G, van den Bergh MR, Veenhoven RH, Wang X, Trzcinski K, Bonten MJ, Rossen JW, et al: Dysbiosis of upper respiratory tract microbiota in elderly pneumonia patients. ISME J 2016, 10:97-108.

39. Zakharkina T, Martin-Loeches I, Matamoros S, Povoa P, Torres A, Kastelijn JB, Hofstra JJ, de Wever B, de Jong M, Schultz MJ, et al: The dynamics of the pulmonary microbiome during mechanical ventilation in the intensive care unit and the association with occurrence of pneumonia. Thorax 2017, 72:803-810.

40. Bousbia S, Papazian L, Saux P, Forel JM, Auffray JP, Martin C, Raoult D, La Scola B: Repertoire of intensive care unit pneumonia microbiota. PLoS One 2012, 7:e32486.

41. Segal LN, Clemente JC, Tsay JC, Koralov SB, Keller BC, Wu BG, Li Y, Shen N, Ghedin E, Morris A, et al: Enrichment of the lung microbiome with oral taxa is associated with lung inflammation of a Th17 phenotype. Nat Microbiol 2016, 1:16031.

42. Kitsios GD, Fitch A, Manatakis DV, Rapport SF, Li K, Qin S, Huwe J, Zhang Y, Doi Y, Evankovich J, et al: Respiratory Microbiome Profiling for Etiologic Diagnosis of Pneumonia in Mechanically Ventilated Patients. Front Microbiol 2018, 9:1413.

43. Carratala J, Mykietiuk A, Fernandez-Sabe N, Suarez C, Dorca J, Verdaguer R, Manresa F, Gudiol F: Health care-associated pneumonia requiring hospital admission: epidemiology, antibiotic therapy, and clinical outcomes. Arch Intern Med 2007, 167:1393-1399.

44. Dickson RP, Schultz MJ, van der Poll T, Schouten LR, Falkowski NR, Luth JE, Sjoding MW, Brown CA, Chanderraj R, Huffnagle GB, et al: Lung Microbiota Predict Clinical Outcomes in Critically III Patients. Am J Respir Crit Care Med 2020, 201:555-563.

\section{Figures}




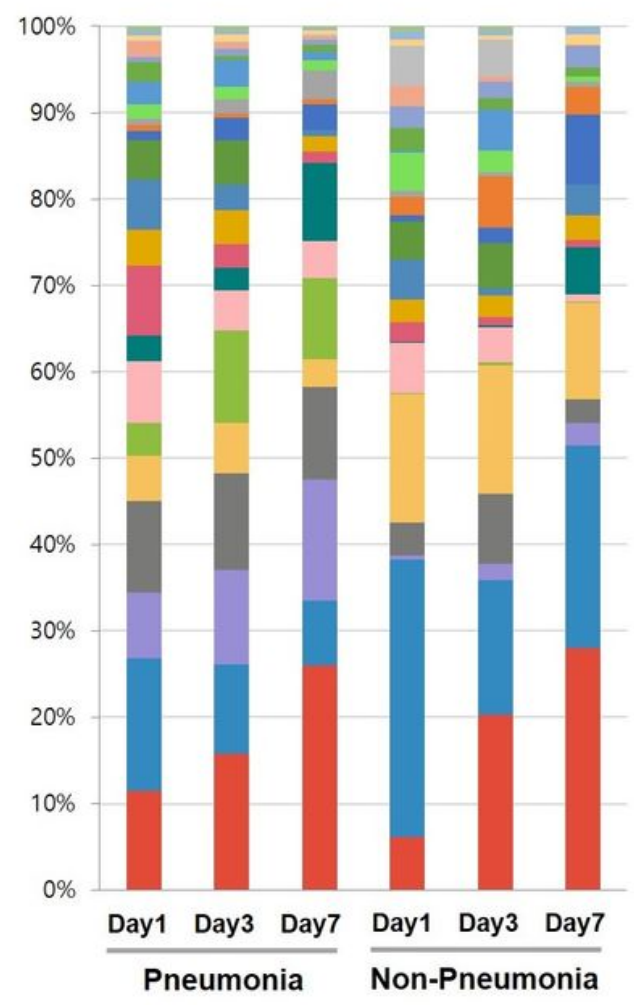

- Campylobacter

- Fusobacterium

Porphyromonas

- Serratia

Actinomyces

- Alloprevotella

- Granulicatella

- Ralstonia

Enterobacter

- Stenotrophomonas

- Lactobacillus

- Mycoplasma

- Escherichia

- Haemophilus

- Veillonella

- Neisseria

- Enterococcus

- Klebsiella

- Pseudomonas

- Prevotella

- Staphylococcus

- Corynebacterium

- Streptococcus

- Acinetobacter

Pneumonia

Non-Pneumonia

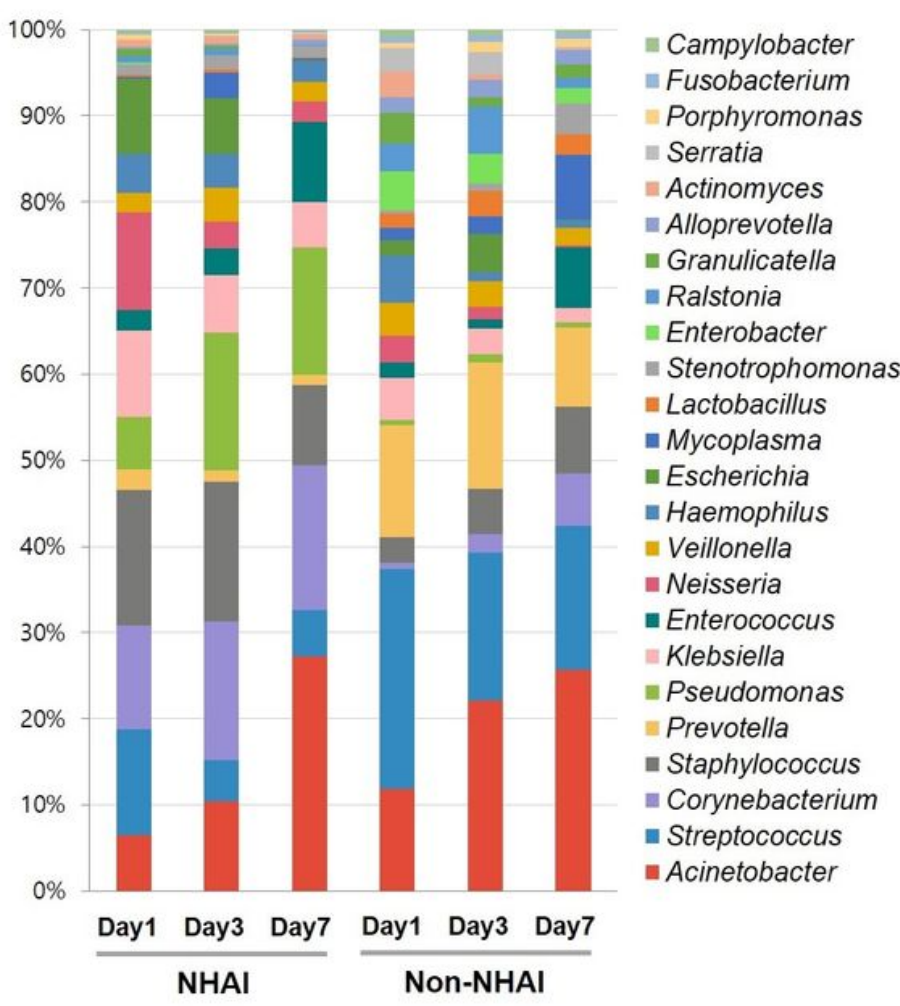

Figure 1

Relative abundance of bacterial communities in the endotracheal aspirates of the study participants over time. Taxonomic summaries of the 180 samples are shown for pneumonia and non-pneumonia patients (a) and for NHAl and non-NHAl patients (b).

\section{Corynebacterium}

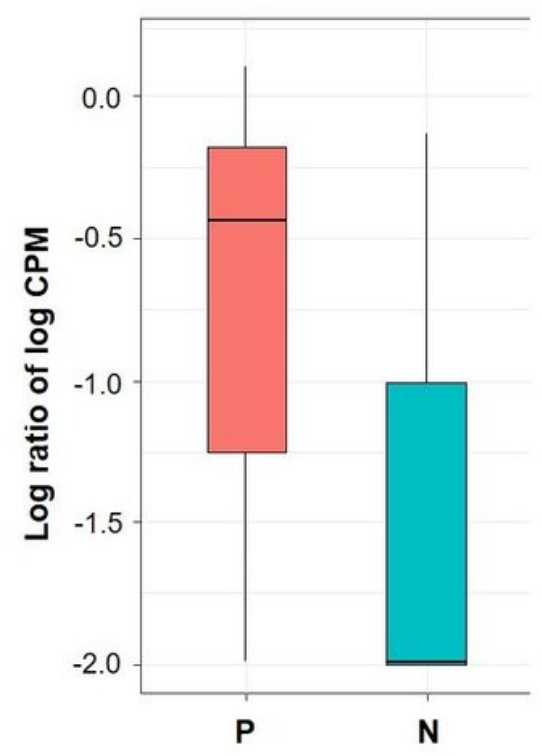

Corynebacterium ulcerans

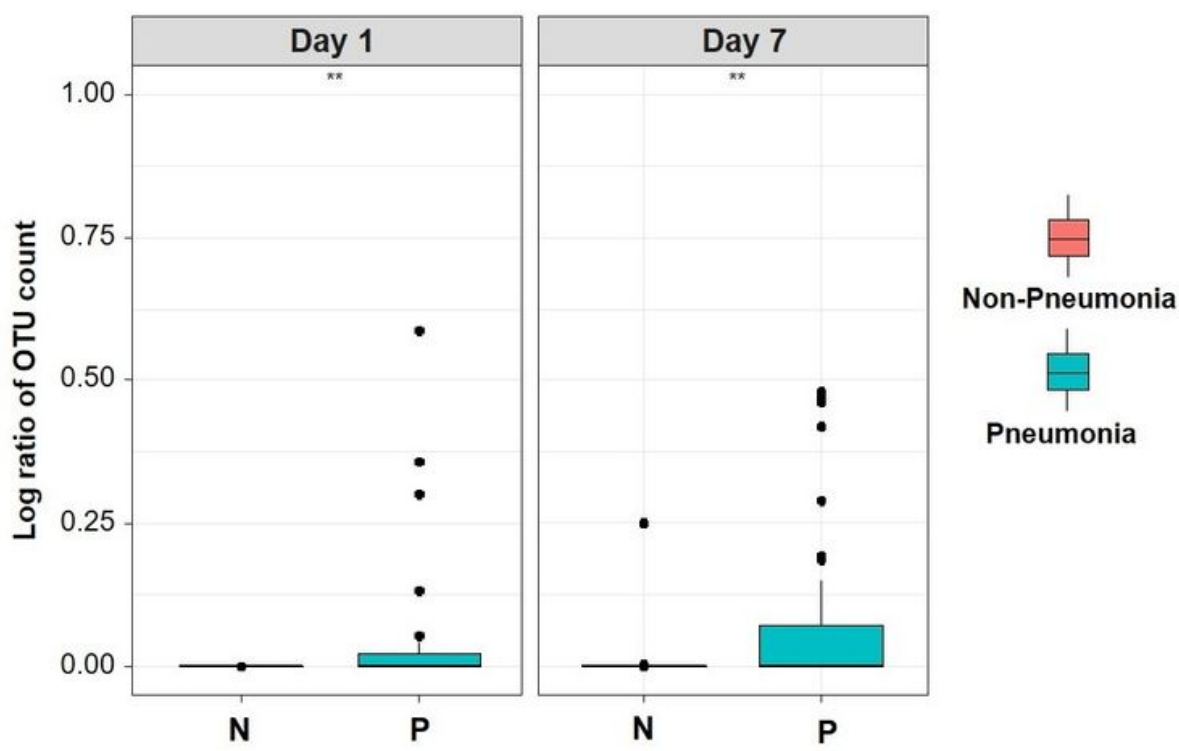

Figure 2 
Increased abundance of Corynebacterium ulcerans in patients with pneumonia. (a) More frequent occurrence of Corynebacterium OTUs in the pneumonia and non-pneumonia groups, respectively. (b) Longitudinal patterns of relative abundance of $\mathrm{C}$. ulcerans OTUs in the pneumonia and non-pneumonia groups.
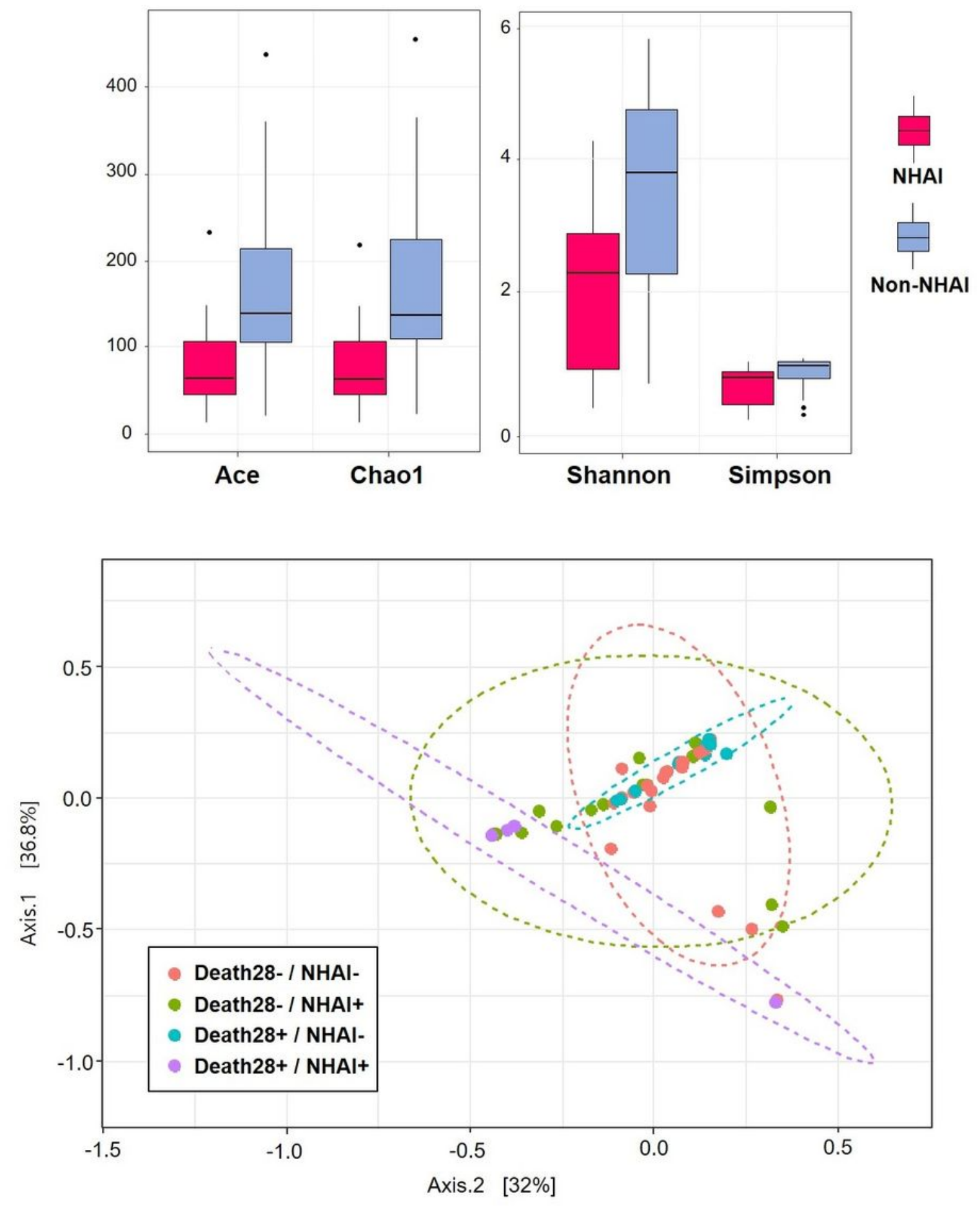

Figure 3

(a) Comparison of the different metrics of a-diversity between the NHAl group (group 1) and non-NHAI group (group 0). (b) Microbial community structure in the endotracheal aspirates according to risk factors 
for NHAI based on weighted UniFrac distances. A PERMANOVA, performed using the Adonis function in the $\mathrm{R}$ package vegan) was conducted to compare the microbial community structure between the NHAl and non-NHAI groups. The analysis was adjusted for age, sex, APACHE score and Charlson Comorbidity Index score.

\section{Supplementary Files}

This is a list of supplementary files associated with this preprint. Click to download.

- Additionalfile1.docx

- AdditionalFile2.docx 